\title{
Data Capture from Medical Device using Augmented Reality
}

\author{
Manimala.G, Kavitha.V, Abinaya.V, Nisha G.Mathur
}

\begin{abstract}
In order to develop medical devices that connect to hand held devices via Bluetooth or USB, a new approach is followed by using Augmented Reality (AR) and machine vision which is utilized to identify digitally a biomedical device and capture reading using Augmented Reality to create 3D imaging of tumors and human organs accurately. By superimposing anatomic structures segmented from tomography images (e.g., CT, MR) on the intraoperative video images. It is integrated with EMR's, clinical information feeds and medical imaging systems to support clinical decision making through a combined AR view. The output will be in the form of labels; $3 D$ rendered models, or shaded modifications. It uses everyday technology - computers, tablets or smart phones with cameras can be used to connect surgeons in real time, anywhere in the world.AR can be used to capture medical device information on a mobile device and can automate the data collection tasks by health workers in all the developing countries.
\end{abstract}

Keywords: Augmented Reality, Electronic Medical Record (EMR),3D imaging

\section{INTRODUCTION}

The Nation's GDP of $3 \%$ is spent on various surgery procedures and development. The factors that are used in determining hospital pricing includes the imaging and lab work-up, medications, operating room instruments, care taken after the surgery and doctor's fees [1]. Any kind of advancement in technology that decreases aggressiveness of the procedure, produces better results and lessens the no. of days spent in the hospital will have a profitable outcome on a given hospital's bottom line. These objectives can be achieved by using the virtual reality (VR) and augmented reality (AR) enhanced surgery [1]. The term "Augmented Reality" (AR) refers to the currently emerging technology which overlays the image that is generated by a computer in the real world from the user's perspective and thereby provides a composite view. A basic requirement of $\mathrm{AR}$ is that the output produced by the computer graphics must be linked to the viewed scene, that is, the position perceived from scene and the computer-generated objects must match.

Revised Manuscript Received on October 12, 2019.

* Correspondence Author

Manimala.G*, Dept. of CSE, Sri Sai Ram Engineering College, Chennai, India. Email: manimala.cse@sairam.edu.in

Kavitha.V, Dept. of CSE, Sri Sai Ram Engineering College, Chennai, India. Email: kavitha.cse@sairam.edu.in

Abinaya.V, Dept. of CSE, Sri Sai Ram Engineering College, Chennai, India. Email: abibe.99@gmail.com

Nisha.G.Mathur, Dept. of CSE, Sri Sai Ram Engineering College, Chennai, India. Email: nishagmathur98@gmail.com
From gaming industries, to surgical procedures, Augmented Reality is widely used because it brings an imaginary world, into the actual real world scenario that helps in improving the things people see, hear and feel. The Augmented reality lies in the middle of the mixed reality i.e., between the imaginary world and the real world [4].,among the various reality technologies.

In augmented reality applications, users see both synthetic and natural light. It is accomplished by superimposing the projected images above a pair of glass or goggles. Moving into the surgical field, we see that a few hundred years ago surgeries were performed by opening up the human body. This caused death due to huge amount of blood loss and infection. Therefore diagnosis and treatments was difficult. Nowadays diagnosis was performed in less invasive ways. With the advancement in the technologies, devices and instruments were slowly invented and developed, which reduced the loss of the lives. Technology has joined hands with the medical field to provide less invasive surgeries like Endoscopy, Laparoscopy, Robot-assisted surgery (robotic surgery) which imparts accuracy in surgeries which thereby reduces the risks and the errors [6].

But still surgeries are in the hands of mental assumption and mental calculation of the surgeons. The displays used for the surgical procedures are mostly located at a large distance from the area of the surgery (Fig.1). This arrangement makes the surgeons to take extra measures to match them with the anatomy of the patient during the surgery. This is a major problem which will cause an interruption in surgical flow. Moreover, most of the therapeutic information in the medical images provided to the surgeons in the form of two dimensional cross sectional images [8]. The surgeon would require reconstructing the 2-D view accordingly into a $3-\mathrm{D}$ view of the image in their mind. However, the perception of the view of the image differs between various doctors. This makes medical errors as the $3^{\text {rd }}$ leading reason for the deaths across the world.

The main aim of using AR during surgery is to augment the doctor's ability to make use of the medical imagery to decrease the effect of surgeries and thereby increasing safety and accuracy.

A head-mounted display (HMD) must be utilized for generating a unrealistic view to help in the process of inspecting the blueprints of plane. The merging of the virtual and real scenarios in the HMD is a very tedious process. 


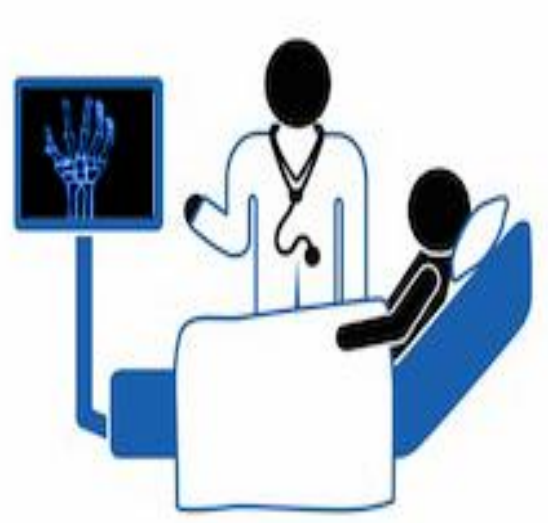

Fig.1: Major drawbacks in today's surgery scanned images are located away from the surgeon field.

The previous medical experience with CAS clearly indicates that it is very difficult to find a place for the very large device such as the HMD in the surgery room. The head-mounted display (HMD) in these types of surgery provides the doctor with a clear view of the surgical field with computer-generated medical images [3]. A HMD has more than one (mostly 2) tiny monitors and semi-transparent mirrors embedded within the spectacles, glasses, helmet or visor [7]. HMD-type image-guided navigation systems have been already in use in various forms, to assist surgeons by supporting and improving visualization of the surgical area during the critical time. With additional improvements to display resolution, structures, and the designs that merge both the real world and registered synthetic imagery have significantly increased the benefits of such systems to medical use [7].

An electronic health record (EHR), or electronic medical record (EMR), is a systematic collection of patient's illness details and medications in a digital format. This information about the patient and his/her medical information can be mutually exchanged across different medical networks. Electronic medical record contains data like personal information about the patients, their illness, symptom for their illnesses, details about previously prescribed medicines, test results, scan reports, $\mathrm{X}$-rays etc..

\section{LITERATURE SURVEY}

In the paper [6], the usage of augmented reality in laparoscopic and robotic surgery is discussed. They used visualization enhancement for interventions such as cholecystectomy, laparoscopy for suspected carcinomatosis, caudal pancreatic resection and robotic liver resection. For better visualization of the structures, intravenous contrast enhancement was required. These images were then manipulated with OsiriX for 3D projection. But the drawback in paper [6] is that both the surgeries are the most complicated surgeries. So introducing new technology with less experience in technical aspect during these surgeries may cause more complications and the surgeries may take hours to complete. The doctors might take more time to understand the projection which may delay the surgical procedures; as a result it may cause more complications.

In the paper [7], "Augmented Reality through Wearable Computing" uses video cameras, audio systems, sensors, infrared beacons for sensing location. The main drawback in this system is that all the additional apparatus are added to the wearable device which makes it bulky and not comfortable during the surgeries. Even though it is interactive and designed to show all the information and details about the overlaid image, it might be clumsy making the view little distractive. Moreover it may not project the image with more importance thereby causing more difficulties in attaining the final goal.

\section{PROPOSED SYSTEM DESIGN}

\section{A. Photogrammetric Caliberation}

The most important problem of bringing a correlation between the 3-D position data to that of the 2-D data was done using the photogrammetric calibration [3]. Some parts of the code were utilized to meet the requirements of the current proposal. The algorithm that performs this type of correlation between 3-D and 2-D data or parameters is referred to as the Projective transformation. The Projective transformation is done by mapping lines to lines and they are represented using a $3 \mathrm{X} 3$ matrix consisting of the homogenous coordinates.

The attributes such as the focal length and the translation transformation must be separated from each other so that the projective transformation is performed. This projective transformation can be done only when the image plane of the optical system and the calibration grid remain scattered and are not parallel to each other.

The effective focal length of the optical system, the lens distortion coefficient, the uncertainty scale factor and the coordinates of the display's centre are the four intrinsic attributes that determine the projective properties of the display

These intrinsic parameters are calculated by positioning a crosshair at the centre of the HMD's field-of-view. Also create or modify present medical device with a live camera to capture the image of the scanning part, so that it sees the same view as in the medical device (Fig.2). The cameras are used to scan and collect the data about the surroundings, which will be used to develop a digital model, so that the required output is produced. It takes the image of the body part which is scanned by the medical device using live camera.

\section{B. Image Superimposing Navigation System}

Image Superimpose Surgery makes use of augmented reality, using which 3D images are overlaid on top of the body of the patient and it is assessed by using it as a reference during surgeries. The primary data of the pre-operative information of the patient were

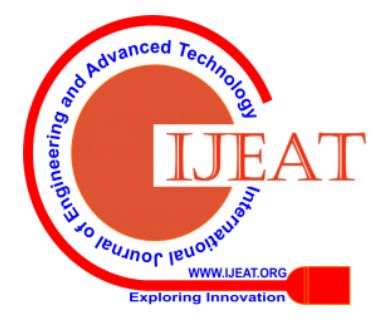


retrieved from the Medical devices and overlaid projected onto the patient's body surface during the operation. For the exact and proper positioning, Head Mounted Device is set to reproduce the augmented elements (Scanned Image) on the patient body surface and positioning the coordinate on the body surface to reduce positional errors.

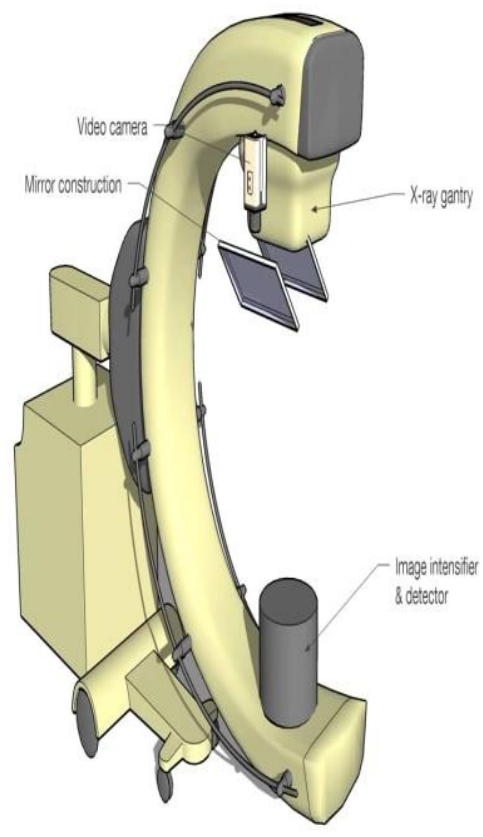

Fig 2: X-Ray machine with additional feature of Video Camera for a live feed

\section{Image Display And Overlay Device Working}

Scanned X-Ray image is projected with the help of a mechanical arm and beamer directly onto the patient to display the virtual and the real-world images (Fig.3). The images that are ready to be projected are created within 20 minutes or less [6]. Landmarks or markers such as the patient's umbilical cord can be used for authorization purpose [6].

The image can be modified accordingly to show different parts of the body such as bone, blood vessels or any other internal organs. This helps to better understand the nature of the patient's organs below the skin and accordingly holes in the skin are made through which the laparoscopic instruments or robotic trocars are placed. These instruments are correctly placed in such a way that a large no. of movements of the instruments are avoided. Ultimately, patient safety is improved. If the anomaly that the doctor is going to perform a very simple operation, then the super imposed images will be very much useful, while there is a requirement for navigation. Moreover, precise dissection through solid organs helps to save as much normal tissue as possible. Along with scanned image superimposed on the skin or any part, the patient details like name, id and scanned image detail can also be included for extra information on the display without disturbing the view of the augmented image.
They help the doctor to identify the patient history without checking the paper works for a long time for simple information.

A head-mounted display is simply a small size monitor that forms a part of the helmet or the data glasses. It is used to gather information of a particular area or field from the user point of view. The military HMDs do not usually display videos or media, but rather give information and tracking as part of a heads-up display for the vehicle being used [7]. The displays in an HMD are most often Liquid Crystal Displays (LCD) [3], though we might come across older models that use Cathode Ray Tube (CRT) displays.

LCD monitors are smaller in size, lightweight, efficient and inexpensive than CRT displays [8]. The two major advantages CRT displays are screen resolution and brightness. Unfortunately, CRT displays are usually bulky and heavy. Almost every HMD using them is either uncomfortable to wear or requires a suspension mechanism to help counteract the weight.

\section{D.Electronic Medical Record System}

Electronic health records are used to store all the patient related information in a clear and easy understandable format. The EMR ensures that the related information of a particular patient is available at all times to all the authorized and the authenticated users at a particular instant. It helps to maintain the patient's record in a safe and secure environment. It avoids the loss of patient's data and errors that may occur due to the misunderstanding of the medicines prescribed by the doctor for a previous illness of the patient. Thus EMR plays a very important role in the medical world.

Some of the main functions of EMR are

- Maintenance of Patient's Illness details

- Lab Result management

- Earlier Patient Care and support

- Administrative processes and reporting

- Reporting and population health [10].

\section{WORKING OF EMR WITH HMD}

Firstly, the medical device and the camera send the data to the computer via Wi-Fi or wire. Wireless access is preferred more than wired, since it is comfortable, covers a larger area. The user's real world interactions are collected using the sensors and they are transferred to get manipulated and interpreted. This helps in identifying body part which is scanned the next time, when it has been interpreted by the device. The data received from the medical device and camera are aggregated and programmed in such a way that, when scanned body is recognized by the Head Mounted Device it replaces or overlaps with the scanned image.

Various devices and the components such as Central processing unit, random access memory, flash memory, Graphical processing unit, GPS etc. that are used in this system require significant computer processing power to execute this process.

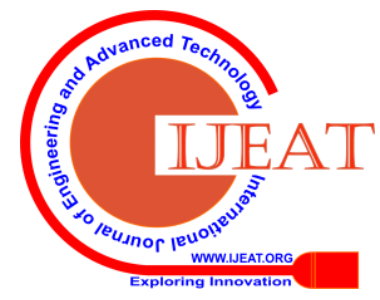


Augmented Reality replaces the original view with the augmented view either fully or partially by superimposing new information on top of it where the graphics images are merged into the video image through graphical overlay rendering.

In the Analysis phase, the recognized picture is analyzed. It retrieves the corresponding scanned image from the system which has been programmed to overlap. The information that is given as input to the camera are manipulated and then they are projected on to the body surface of the patient followed by the process of projection onto a surface in front of the user.

\section{USAGE OF EMR TO THE SOCIETY}

The safe maintenance of digital data regarding the illnesses of the people and the safety of those health related data is the major concerns of the companies to incorporate into their software systems of EMR [10]. The reduction in the number deaths caused due to medical errors helps both the society and the doctor.

An electronic medical health records helps to provide the clear information regarding the prescribed medicine to the patients, thereby reducing the hand transcribed errors. The issues occurring due to lost or misplaced patient files are also eliminated.

In addition to it, EMR and patient care are closely associated with each other such that they help to place the constraints to access the health related data of the patient from the end user's side. Such type of safety has to be taken in to account while maintaining the privacy of the highly confidential patient medical details [10].

\section{EXPERIMENTAL RESULT}

From the experimental result, the scanned image can be overlapped and seen in all the three dimensions. The Fig 3 shows the normal photograph of the hand while scanning. In Fig 4, the augmented data is seen. The scanned image is overlapped on the hand (front side of the hand). In Fig 5, the scanned image is overlapped in the back side of the hand. This helps the doctor to see the scanned image in all 3 dimensional views, to get the insight of the scanned image with accuracy in locating the fracture or tumor. This is achieved using Unity $3 \mathrm{D}$ and Vuforia.

The Unity 3D is the tool which augment the data on to the real world. It positions the target image and Quad image in the same plane which helps to overlap the scanned image (Quad) on the hand (target image). Vuforia acts as the database for the tool which stores all the images and connects the images with the corresponding scanned image without any mismatch using different keys to differentiate.

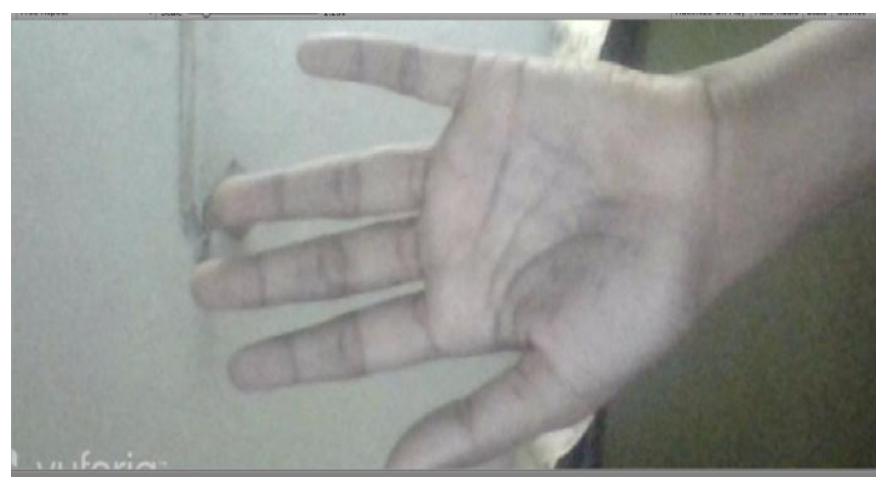

Fig3: The normal photography of the hand while taking the x-ray.

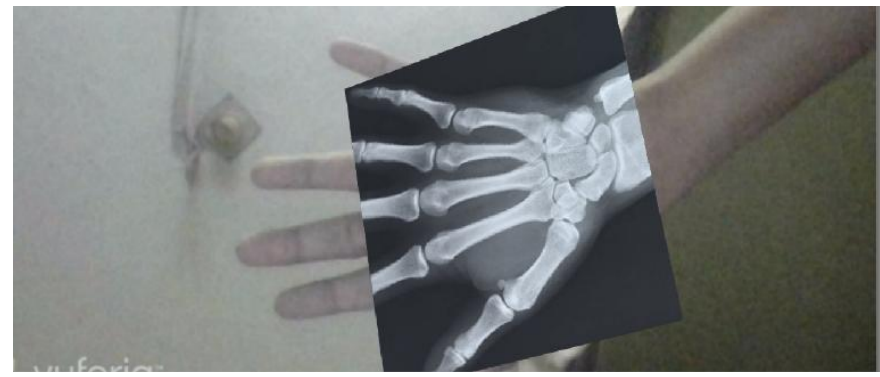

Fig 4: Overlapping the scanned image on the hand (front side of the hand)

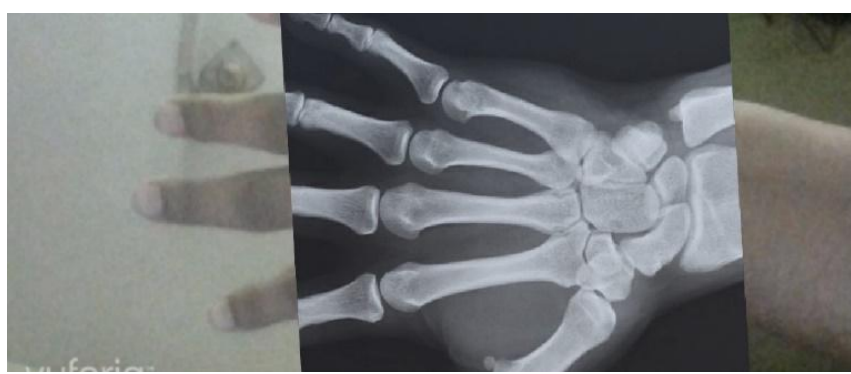

Fig 5: overlapping the scanned image on the hand (Back side of the hand)

\section{CONCLUSION AND FUTURE ENHANCEMENT}

This proposed work overlays the scanned images on the corresponding body part of the patient. Using the marked based augmented reality, the device detects the marker and overlay the corresponding scanned images. This gives the doctors a clarity and accuracy in locating the fractures, tumors and differentiation between healthy and unhealthy tissues. Since the scanned images are directly superimposed on the body part, locating fractures is easy and also the tumor tissues can be differentiated by colored images which are superimposed on the real tissues.

The doctor or patient knows about the exact location and the intensity of the tumor of fracture in the scanned image since it exactly places the scanned image on the patient. The Augmented Reality device has no restriction of movement or space. The images moves or rotates along

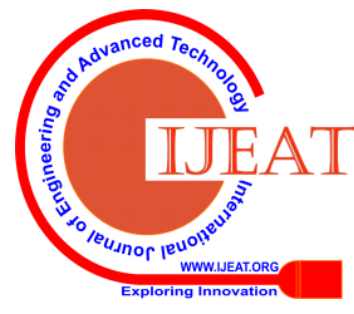


with our head movement. This can be a boon for the future surgeries and many lives can be saved.

In the future enhancement, we will be adding the patient details, case details and the other details of the scanned images. Also store this information in the hospital database. A high resolution camera can also be used for high quality of the images to increase the clarity. This might help the doctors and the patient to have a digital copy of all the reports and images which can be used in the future.

Since the healthcare is still in the hands of mental assumption and paper works, the world's $3^{\text {rd }}$ leading reason for death is medical errors. In India nearly 3 million people die due to such type of errors. Still surgeries are done with mental calculation with no accuracy as the scanned images are in different places rather than in the place of operation. This gives a huge distraction to the doctor during operation. Moreover there is no accuracy in locating the fractures, tumors etc., and it is also difficult to differentiate it from the healthy tissues. This makes doctor to cut the healthy tissue along with the tumor or leave some tumor tissues which may lead to many complications and might end up in doing more surgeries in future.

An EMR is alleged to make the process of patient record-keeping easier, correct, comprehensive and efficient. This helps doctor to maintain the patient's medical information in a digital format such that it becomes easily available to them whenever they are required. Doctors can use any computing device such as a laptop or a desktop to scroll through patients' charts and record notes. Augmented reality techniques will support the surgeons to have better perception, helping them to complete the surgery in an easier, faster and probably safer way. AR is a game changer in the health care field.

\section{REFERENCES}

1. F. Wenzel, F. Recchi, T. Rohlfing, C. M. Brown, R. S. Bakos, R. J. Maciunas, and A. Bani-Hashemi, C. R. Maurer Jr., F. Sauer, B. Hu, B. Bascle, B. Geiger, "Augmented reality visualization of brain structures with stereo and kinetic depth cues: System description and initial evaluation with head phantom," in Proc. SPIE, K. S. Mun, Ed., 2001, vol. 4319, Medical Imaging 2001: Visualization, Display, and Image Guided Procedures, pp. 445-456.

2. Azuma. R. T., "A survey of augmented reality," Presence: Tele operators Virtual Environ., vol. 6, no. 4, pp. 355-386, 1997.

3. L. Preston \& S. Bangay, "An investigation into factors influencing immersion in interactive virtual reality environments," Stud. Health Technol. Inform., vol. 58, pp. 43-51, 1998.

4. S. L. Tang, Chee-KeongKwoh and Keck Voon Ling "Augmented reality systems for medical applications.

5. LanceLiotta, David B Douglas, Clifford A Wilke, David Gibson, Emanuel F Petricoin, "Virtual reality and augmented reality: Advances in surgery"

6. FrancescoVolonté,FrançoisPugin,PascalBucher,Maki Sugimoto "Augmented reality and image overlay navigation with OsiriX in laparoscopic and robotic surgery"

7. "Augmented Reality Through Wearable Computing" Thad Starner, Steve Mann, Bradley Rhodes, Jeffrey Levine Jennifer Healey, Dana Kirsch, Roz Picard, and Alex Pentland.

8. Salb T, Brief J, Burgert O, Gockel T, Hassfeld S, et al. (2002) Intraoperative augmented reality for craniofacial surgery: the INPRES system.

9. Edwards PJ, King AP, Maurer CR, Jr., de Cunha DA, Hawkes DJ, et al. Design and evaluation of a system for microscope-assisted guided interventions (MAGI). IEEE Trans Med Imaging 19: 1082-1093.
10. Nicholas A. Kalogriopoulos, Jonathan Baran, Amit J. Nimunkar and John G. Webster, Electronic Medical Record Systems for Developing Countries

\section{AUTHORS PROFILE}

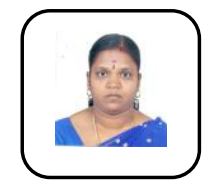

G.Manimala, Assoc.Prof., Dept. of Computer Science and Engineering. Doing Research in the domain of Cloud Computing. Life member of ISTE and MCSIT. Published papers in various International conferences. Scopus indexed journals , National and

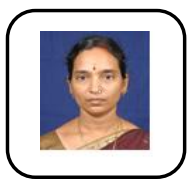

V.Kavitha, Assoc.Prof., Dept. of Computer Science and Engineering. Doing Research in the domain of Service Oriented Architecture. Life member of ISTE and MCSIT. Published papers in International conferences various Scopus indexed journals, National and

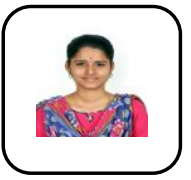

V.Abinaya, Student ., Dept. of computer science and Engineering. Doing her Bachelor Degree Computer Science and Engineering. Member of CSI. Published papers in National and International conferences.

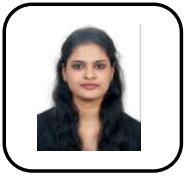

Nisha G.Mathur, Student ., Dept. of Computer science and Engineering. Doing her Bachelor Degree Computer Science and Engineering. Member of CSI. Published papers in National and International conferences. 